\title{
Targeting of "high anthocyanin" white clovers for increased condensed tannin levels via genetic modification
}

\author{
K. RICHARDSON, D. MAHER, G. COUSINS' ${ }^{1}$, J. FORD ${ }^{1}$, K. HANCOCK, A. SCOTT, \\ S. RASMUSSEN and V. COLLETTE \\ Pastoral Genomics, Clo Forage Biotechnology, ${ }^{1}$ Forage Improvement, AgResearch Ltd, Grasslands Research Centre, \\ Palmerston North, New Zealand \\ kim.richardson@agresearch.co.nz
}

\begin{abstract}
White clover cultivars with enhanced levels of foliar condensed tannins would significantly contribute to pasture productivity via increased production, forage quality and decreases in adverse environmental impacts. Recent developments suggest it is feasible to achieve this via expression of a single transgene, but that accumulation will only occur in specialised cell types which contain an active anthocyanin pathway. In this study we: (1) investigated the transformation potential of clover genotypes expressing foliar anthocyanins and (2) introduced two condensed tannin biosynthetic genes into selected genotypes. Decreased anthocyanin accumulation was observed in plants expressing either transgene suggesting a potential change in flux from the anthocyanin to the condensed tannin pathway. These plants represent the first description of genetic transformation in anthocyanin expressing white clover genotypes. Our ultimate aim is to genetically enhance foliar condensed tannin levels in white clover allowing the development of novel "bloat-safe" cultivars conferring improved animal health performance. Results to date represent a significant milestone toward this goal.
\end{abstract}

Keywords: Trifolium repens, condensed tannin, anthocyanin

\section{Introduction}

An ability to produce white clover cultivars with enhanced levels of foliar condensed tannins (CTs) has been a long term aim of pastoral research. High levels of CTs in forage plants can significantly enhance pasture productivity through i) improving animal performance and productivity by increased dietary nitrogen utilisation and reduced occurrence of pasture bloat (Aerts et al. 1999; Barry \& McNabb 1999; Li et al. 1996); ii) improving animal health by reducing internal parasite load (Niezen et al. 1993, 1995) and iii) reducing the emission of greenhouse gases such as methane from farm animals (Woodward et al. 2001).

The genetic regulation of CT biosynthesis is complex. They are formed from the flavonoid biosynthetic pathway which is also involved in the synthesis of flavonols, isoflavonoids and anthocyanins. The CT pathway is a branch of the anthocyanin pathway and comprises an array of structural and regulatory genes for the production of individual precursor units which are polymerised into CTs before transportation into the vacuole (a cellular storage organelle). White clover CTs are produced mainly in floral tissue (Corkhill 1971) while in leaves they are localised to trichomes at levels which are less than the optimum required to confer beneficial animal and environmental impacts.

It has recently become apparent that $\mathrm{CT}$ accumulation is programmed to occur only in specialised cell types (Sharma \& Dixon 2005). Epicatechin, a major building block of CTs, is synthesised directly from anthocyanidin which is also the immediate precursor for formation of anthocyanin pigments (Xie et al. 2003). Therefore, to introduce CTs into tissues where they do not naturally occur, it is necessary to activate the anthocyanin pathway from which the precursors of CTs are derived (Dixon et al. 2005). Activation of the anthocyanin pathway in tobacco and Arabidopsis was achieved by the transgenic expression of a transcription factor (a gene which regulates the expression of other genes) called PAP1 (Sharma \& Dixon 2005). By the subsequent transgenic expression of both PAP1 and the CT structural gene BANYULS (BAN) it was possible to produce CTs in tobacco leaves (Xie et al. 2006).

As an alternative to introducing all of the necessary upstream anthocyanin and downstream CT genes by genetic transformation, a simpler approach of using cultivars with natural accumulations of foliar anthocyanin may provide the appropriate cell types and functioning regulatory and structural genes. Such an approach offers the potential for $\mathrm{CT}$ accumulation following expression of either of the CT structural genes, BAN or leucoanthocyanidin reductase (LAR) via genetic transformation. A similar strategy using Medicago truncatula lines which accumulate foliar anthocyanins resulted in a reduction in anthocyanin and an accumulation of oligomeric CTs in the foliage following ectopic expression of BAN (Xie et al. 2006).

In this study we have used genotypes accumulating foliar anthocyanin (High Anthocyanin White Clover, HAWC) obtained from a wide range of AgResearch germplasm. Genotypes possessing a variety of 
Table 1 HAWC genotype responses to Agrobacterium-mediated genetic transformation.

\begin{tabular}{clccc}
\hline Accession \# & Description & $\begin{array}{c}\text { No. transformation } \\
\text { experiments }\end{array}$ & $\begin{array}{c}\text { Plants/ } \\
\text { explants }\end{array}$ & $\begin{array}{c}\text { Transformation } \\
\text { frequency (\%) }\end{array}$ \\
\hline $\begin{array}{c}\text { Huia control } \\
\text { C15803 }\end{array}$ & Homozygous-dominant Feathermark Huia & 1 & & \\
Ornamental & & & $4 / 250$ & 1.6 \\
C19255 & Non-nodal multifoliate ornamental (F3) & 1 & $7 / 250$ & 2.8 \\
C23697 & Multifoliate Purple-pink flowering ornamental polycross & 2 & $0 / 500$ & 0 \\
C19008 & Multifoliate ornamental non-nodal paircross & 1 & $9 / 250$ & 3.6 \\
C12199 & Ornamental paircross & 2 & $0 / 500$ & 0 \\
C19250 & Non-nodal multifoliate ornamental (F3) & 2 & $14 / 500$ & 2.8 \\
C18988 & Multifoliate ornamental paircross & 1 & $0 / 250$ & 0 \\
C21558 & Crimson leaf ornamental/Pink-purple flowers & 2 & $7 / 450$ & 1.6 \\
C21557 & Crimson leaf ornamental/Red flowers & 2 & $60 / 500$ & 12 \\
Agronomic & & & & \\
$25179 \mathrm{dxA}$ & Red V/Feathermark Huia x Aran & 12 & $39 / 3008$ & 1.8 \\
25179dxT & Red V/Feathermark Huia x Tribute & 10 & $41 / 2500$ & 2.0 \\
\hline
\end{tabular}

anthocyanin leaf markings from both ornamental and agronomic types were first tested for their potential in genetic transformation and then cloned DNA fragments designed for transgenic expression of BAN and LAR were introduced into selected genotypes. Results indicate that these genotypes are readily transformed and introduction of BAN or LAR frequently results in a reduction of foliar anthocyanin suggesting a redirection of the flavonoid pathway from anthocyanins in favour of the condensed tannin pathway.

\section{Methods}

\section{Germplasm}

HAWC genotypes obtained from the Margot Forde Germplasm Centre were selected based upon a distribution of anthocyanin in leaves ranging from complete coverage of the leaf surface to minor markings and were characterised as either ornamental or agronomic types (Table 1).

\section{Agrobacterium strain and binary vectors}

The binary vector pHZBar::intGUS was used to determine the transformation potential of HAWC genotypes. This vector contains the bar gene which confers resistance to the herbicide ammonium glufosinate to allow selection of transformed cells and the GUS reporter for simple histochemical identification of transformed tissues. Expression of both genes is driven by a $35 \mathrm{~S}$ promoter from the Cauliflower Mosaic Virus. Similarly, genomic clones of the BAN and LAR genes, isolated from white clover, were cloned in place of the GUS gene. For transformation, cultures of the avirulent Agrobacterium tumefaciens strain GV3101 containing the appropriate vectors were grown in MGL broth overnight on a rotary shaker at $28^{\circ} \mathrm{C}$, harvested by centrifugation and resuspended in a solution of $10 \mathrm{mM}$ $\mathrm{MgSO}_{4}$.

\section{White clover transformation}

Agrobacterium-mediated transformation into white clover used a procedure adapted from Voisey et al. (1994). Briefly, cotyledons of sterile, imbibed seeds were dissected for transformation. An aliquot of Agrobacterium suspension was dispensed to each dissected cotyledon, and co-cultivated for 72 hours. Transformed cotyledons were transferred to a regeneration medium supplemented with ammonium glufosinate $(5 \mathrm{mg} / \mathrm{L})$. Whole rooted shoots regenerating from cotyledonary explants under ammonium glufosinate selection were transferred to the greenhouse. Transformation frequencies were calculated as the number of transformed plants generated per one hundred explants as confirmed by PCR analysis.

\section{PCR analysis}

Putatively transformed plants were analysed for transgene integration by the polymerase chain reaction (PCR). Genomic DNA was isolated from plants using the Qiagen DNeasy Plant Mini Kit and plants were screened for the presence of the octapine synthase termination signal (ocs3') which results in amplification of a 439bp fragment internal to ocs3' sequence.

RT-PCR was used to identify plants actively expressing the BAN and LAR transgenes. Total RNA was isolated from mature leaf samples using the Qiagen RNAeasy Mini Kit. Complementary DNA was prepared using the Invitrogen SuperScript III System and used for RT-PCR reactions as per the manufacturer's instructions. Amplification gave a product size of 540bp for the 35S/ BAN cDNA product and a product size of $513 \mathrm{bp}$ for the 35S/LAR.

\section{Anthocyanin extraction and measurement}

For each plant, the leaves were harvested along the length of two separate stolons. The position of each leaf was 
Figure 1 Relative anthocyanin levels measured from leaf tissue of plants expressing BAN (AC4603 and AC4702) and LAR (AC4802 and AC4804) grown under greenhouse conditions. The youngest (1) to the oldest (4) leaflets were taken from two independent stolons. The average Anthocyanin Index for the non-transformed controls is shown in grey while the transformed plants are in black.
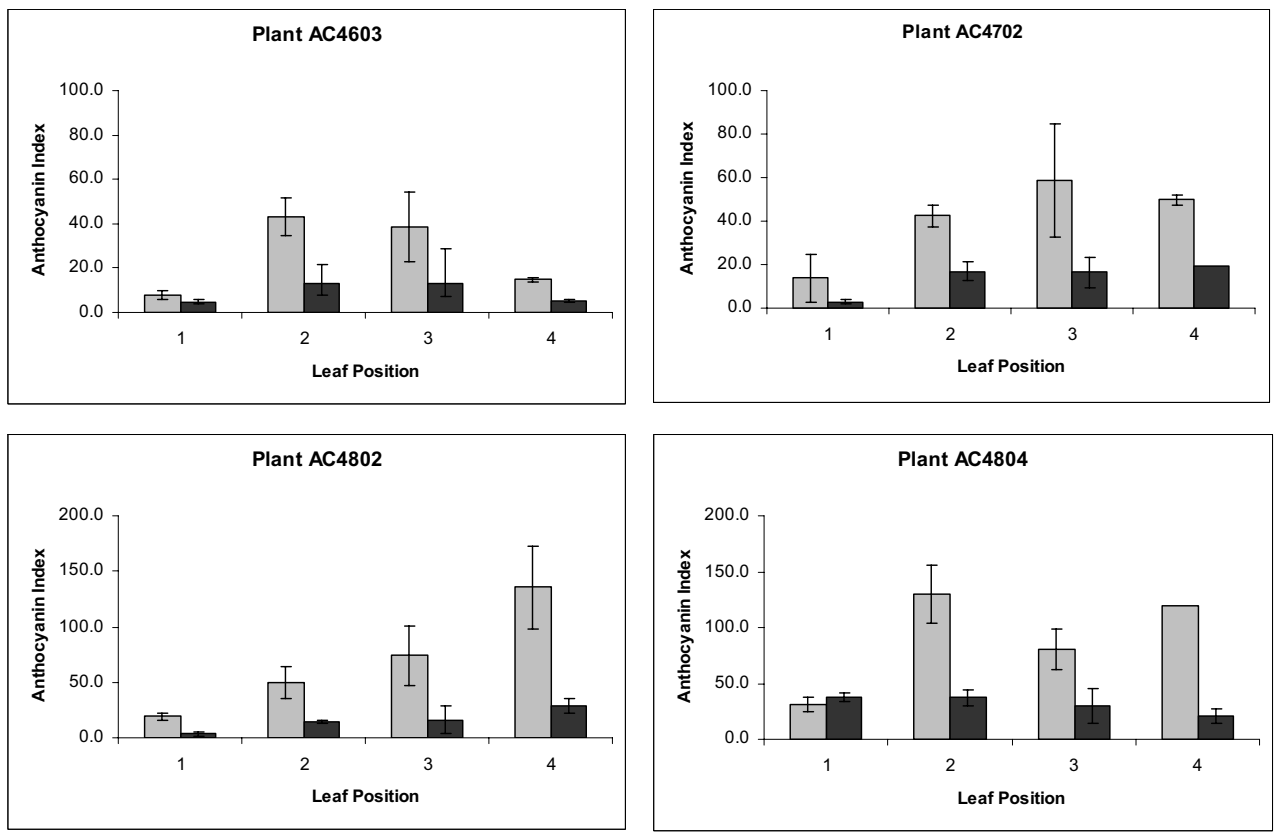

recorded from 1 , the youngest to 4 the oldest. Anthocyanins were extracted overnight in methanol acidified with $1 \% \mathrm{HCl}$ followed by a chloroform wash. Anthocyanins were removed from the aqueous layer and quantified by spectrophotometry at an absorbance of 530 and $657 \mathrm{~nm}$. The relative amount of anthocyanin per $\mathrm{mg}$ fresh weight was calculated from the $A_{530}$ and $A_{657}$ measurements.

\section{Results}

\section{Determination of transformation potential in HAWC}

Stable transformation of plants with pHZBar::intGUS was achieved for all but three of the HAWC genotypes assessed (Table 1). The control genotype C15803 performed in a similar manner to that expected for the cultivar Huia where a transformation frequency of $1.6 \%$ was obtained. Transformation frequencies for genotype C21557 were significantly higher. An average frequency of $12 \%$ was achieved and was consistent for both of the transformation experiments performed and is approximately five-fold higher than the transformation frequency typically obtained for Huia.

Development of anthocyanin pigments was observed following transformation of genotypes C21557, C21558 and C23697. Each of these genotypes displayed a strong pigmentation in leaves, flowers and seed and pigmentation was easily visible on cotyledons 2 to 3 days after inoculation with Agrobacterium. Anthocyanin leaf marks were not frequently observed in vitro but this was not unexpected due to the low light/low stress tissue culture environment. However, natural anthocyanin of leaf marks developed when plants were transferred to the greenhouse.

\section{Anthocyanin responses in HAWC ectopically expressing BAN or LAR}

Following successful transformation of HAWC with the GUS reporter gene, selected genotypes (C19250, C21558, C21557, 25179d x A, 21579d x T) were used for the introduction of genetic constructs designed for the ectopic expression of BAN or LAR. In order to reduce variations due to genetic background, plants were generated in clone pairs, that is, a transformed and nontransformed plant derived from the same cotyledon explant. This allowed a comparison of anthocyanin levels in response to expression of the transgene.

A marked reduction in anthocyanin was observed coincident with transgene expression. For paired plants the relative quantity of anthocyanin was approximately two thirds lower in those plants expressing the transgene compared to the non-transformed controls (Fig. 1).

\section{Discussion}

Transformation of legumes is typically genotype dependant. That is, only a specific genotype or cultivar performs well enough to be useful for generation of 
transformed plants. Therefore, the identification of suitable genotypes from a wide genetic base which possess good characteristics for plant transformation provides a major asset for gene function testing.

Here we have demonstrated successful genetic transformation of HAWC which display a distribution of foliar anthocyanin ranging from complete coverage of the leaf surface to minor markings using a standard protocol previously optimised for white clover. In general, high levels of anthocyanin accumulation correlate with poor agronomic performance (D Woodfield pers. comm.). An exception is the Feathermark red mid-rib anthocyanin pattern. This mark has been stabilised in accession C15803 (Huia) which was used here as a control genotype for transformation. Another exception is the Red V. Both occur as single dominant alleles. The agronomic types used here are the result of combining both the feathermark and Red V which were then backcrossed to elite cultivars Aran and Tribute to produce a segregating population.

Ectopic expression of clover BAN and LAR caused an observable reduction of anthocyanin in leaves. The reduction in anthocyanin suggests a diversion in the pathway away from anthocyanin production and towards $\mathrm{CT}$ accumulation. However, no CTs were detected in the leaf tissue either by DMACA staining or the highly sensitive LC-MS method (data not shown). Our LCMS results could not detect the presence of the CT monomers epicatechin or catechins or oligomers, within the limits of analysis ( $4 \mu \mathrm{g}$ catechin $/ \mathrm{ml})$. As with our observations in white clover, similar reductions in anthocyanin have been described in M. truncatula and tobacco transformed with BAN (Xie et al. 2006). Levels of CT in M. truncatula leaves was measured by thin layer chromotography and were reported to increase threefold compared to wild type but this was due to monomer and small oligomer units rather than the expected CT polymers. As with Medicago, white clover already containing anthocyanins in leaf tissue will also possess the appropriate cell types and the upstream genetic machinery. The disparity in the results suggests we have to fine tune the approach to boost CT production in clover leaves. It may be possible, given the CT profile of white clover, that both BAN and LAR must be expressed simultaneously to effect a shift in CT production.

\section{ACKNOWLEDGEMENTS}

This work was supported by Pastoral Genomics, an industry-good organization funded by the Foundation for Research Science \& Technology, Fonterra, Meat \& Wool New Zealand, Dairy NZ, DEEResearch and AgResearch.

\section{REFERENCES}

Aerts, R.J.; Barry, T.N.; McNabb, W.C. 1999. Polyphenols and agriculture: beneficial effects of proanthocyanidins in forages. Agriculture, Ecosystems and Environment 75: 1-12.

Barry, T.N.; McNabb, W.C. 1999. The implications of condensed tannins on the nutritive value of temperate forages fed to ruminants. British Journal of Nutrition 81: 263-272.

Corkhill, L. 1971. Leaf markings in white clover. Journal of Heredity 62: 307-310.

Dixon, R.A.; Sharma, S.B.; Xie, D. 2005. Proanthocyanidins - a final frontier in flavonoid research? New Phytologist 165: 9-28.

Li, Y.G.; Tanner, G.; Larkin, P. 1996. The DMACA$\mathrm{HCl}$ protocol and the threshold proanthocyanidin content for bloat safety in forage legumes. Journal of the Science of Food and Agriculture 70: 89-101.

Niezen, J.H.; Waghorn, T.S.; Waghorn, G.C.; Charleston, W.A.G. 1993. Internal parasites and lamb production - a role for plants containing condensed tannins? Proceedings of the New Zealand Society for Animal Production 53: 235-238.

Niezen, J.H.; Waghorn, T.S.; Charleston, W.A.G.; Waghorn, G.C. 1995. Growth and gastrointestinal nematode parasitism in lambs grazing either lucerne (Medicago sativa) or sulla (Hedysarum coronarium) which contains condensed tannins. Journal of Agricultural Science 125: 281-289.

Sharma, S.B.; Dixon, R.A. 2005. Metabolic engineering of proanthocyanidins by ectopic expression of transcription factors in Arabidopsis thaliana. The Plant Journal 44: 62-75.

Voisey, C.R.; White, D.W.R.; Dudas, B.; Appleby, R.D.; Ealing, P.M.; Scott, A.G. 1994. Agrobacterium-mediated transformation of white clover using direct shoot organogenesis. Plant Cell Reports 13: 309314.

Woodward, S.L.; Waghorn, G.C.; Ulyatt, M.J.; Lassey, K.R. 2001. Early indications that feeding Lotus will reduce methane emission from ruminants. Proceedings of the New Zealand Society for Animal Production 61: 23-26.

Xie, D.; Sharma, S.R.; Paiva, N.L.; Ferreira, D.; Dixon, R.A. 2003. Role of anthocyanidin reductase, encoded by BANYULS in plant flavonoid biosynthesis. Science 299: 396-399.

Xie, D-Y.; Sharma, S.B.; Wright, E.; Wang, Z-Y.; Dixon, R.A. 2006. Metabolic engineering of proanthocyanidins through co-expression of anthocyanidin reductase and the PAP1 MYB transcription factor. The Plant Journal 45: 895-907. 